\title{
Edad y crecimiento del pez Haemulon steindachneri (Perciformis: Haemulidae) en el suroeste de la isla de Margarita, Venezuela
}

\author{
Misael Morales ${ }^{1} \&$ Leo W. González ${ }^{2}$ \\ 1. Escuela de Ciencias Aplicadas del Mar, Universidad de Oriente, Núcleo Nueva Esparta, Venezuela; \\ misaeldm@hotmail.com \\ 2. Área de Biología y Recursos Pesqueros, Instituto de Investigaciones Científicas, Universidad de Oriente, Núcleo \\ Nueva Esparta, Venezuela; lwgc25@gmail.com
}

Recibido 23-III-2009. C Corregido 02-VIII-2009. Aceptado 02-IX-2009.

\begin{abstract}
Age and growth of the grunt, Haemulon steindachneri (Perciformis: Haemulidae), from the Southwest area of Margarita Island, Venezuela. Studies on fish age and growth are essential to establish models on population dynamics. We determined age and growth of the grunt Haemulon steindachneri from the Southwest Margarita Island using sagita otoliths (direct method) and length frequency (indirect method). A total of 953 individuals were captured by the handcrafted fleet from Boca del Río (Margarita Island) between July 2005 and June 2006. The length-weight relation was established and growth parameters from both curves (length and weight) were estimated using the von Bertalanffy model. The analysis of the annual rings in otoliths established four age groups, and group $3^{+}$was the most representative, with $44.5 \%$ of the sample. The calculation of marginal increases of otoliths suggested the development of an annual ring in April, which coincides with the maximum reproduction period. There were no significant differences in the slopes of the curves $b$ $\left(\mathrm{t}_{\mathrm{s}}=-1.81 ; \mathrm{p}>0.05\right)$ and the intercepts $a\left(\mathrm{t}_{\mathrm{s}}=-1.17 ; \mathrm{p}>0.05\right)$ of females and males. Length-weight for both sexes was $\mathrm{W}=0.0003 * \mathrm{~L}^{2.89}$, which indicates an alometric growth pattern. Growth parameters were established as: $\mathrm{L}_{\infty}=231 \mathrm{~mm}, \mathrm{~W}_{\infty}=203 \mathrm{~g}, \mathrm{k}=0.569$ year $^{-1}$ (direct method) and $\mathrm{L}_{\infty}=228 \mathrm{~mm}, \mathrm{~W}_{\infty}=195 \mathrm{~g}, \mathrm{k}=0.580$ year $^{-1}$ (indirect method), which suggests that it is a short life species that grows moderately rapidly. The index of growth phi prime ( $\left.\varnothing^{\prime}\right)$ coefficient of variation $(\mathrm{CV})$, showed similarity between the growth parameters obtained by both (direct and indirect) methods. The application of the indirect method is recommended, as well as the periodic analysis of population parameters. Rev. Biol. Trop. 58 (1): 299-310. Epub 2010 March 01.
\end{abstract}

Key words: age, growth, Haemulon steindachneri, Margarita Island, Venezuela.

Los estudios sobre edad y crecimiento de peces son esenciales en la evaluación pesquera y en los modelos de dinámica de poblaciones, tanto en los trópicos como en cualquier otra zona. La relación entre la edad y el crecimiento con la abundancia, a menudo se usa para determinar la producción íctica y, por lo tanto, son factores importantes en la gestión o manejo de las pesquerías (Pauly 1983, Morales 1991, Pascual 1996). Es importante conocer la edad en la que una especie alcanza la longitud adecuada para la pesca y su reclutamiento a la pesquería, así como el crecimiento, mortalidad y otros parámetros fundamentales de una población, para la aplicación en la regulación pesquera (Tresierra \& Culquichicon 1993).

La edad de muchas especies puede determinarse a partir de las marcas de crecimiento presentes en algunas estructuras duras, como escamas, otolitos, cleitra, urohiales, vértebras, espinas y radios (Holden \& Raitt 1975, Tavares 2001, Araya \& Cubillos 2002), que se forman debido a factores intrínsecos, como el desove, metabolismo y cambios de residencia, o ambientales, como temperatura, salinidad, luz y alimento (González 1977, 1979). Por otra 
parte, muchos peces viven en un ambiente tan uniforme que prácticamente no se forman discontinuidades en sus estructuras esqueléticas, por lo que la edad debe ser determinada mediante métodos indirectos como el análisis de la frecuencia de tallas.

González (1977) indica lo complicado que puede ser el uso de otolitos en peces tropicales, por la dificultad en la identificación de los anillos verdaderos y falsos. Sin embargo, cabe resaltar que actualmente se han mejorado las técnicas utilizadas para la lectura de los otolitos, lo cual incrementa la confiabilidad de las mismas (Morales 1991). Las escamas y otolitos son las estructuras duras más utilizadas en estudios de edad y crecimiento en peces óseos. En el caso de las escamas, por su facilidad en la remoción y almacenaje. Otra estructura como el cleitrum (hueso de la cintura escapular), muestra claras marcas de crecimiento en muchas especies, pero su uso no es tan difundido, debido en parte, a que su preparación implica una buena limpieza que requiere la extracción total del tejido muscular (Holden \& Raitt 1975, Eslava 1991).

En Venezuela, Eslava (1991) y Barreto (1996), han realizado estudios de edad y crecimiento del pez, H. steindachneri a través del análisis de estructuras duras. Igualmente, se ha demostrado la validez de esta metodología en otras especies como Sardinella aurita (Heald \& Griffiths 1967, González 1985), Cynoscion sp (González 1977), Eugeres plumieri (Romero \& González 1986), Lutjanus analis (Palazón \& González 1986), Opisthonema oglinum (González \& Mengual 1995) y Lutjanus purpureus (González et al. 1998, González \& Eslava 1999).

H. steindachneri es una especie íctica que forma cardúmenes, generalmente acompañada de otras especies de Haemulónidos (Humann \& Deloach 2002). En la isla de Margarita, es capturado principalmente con nasas, anzuelos y mandingas, y su pesca no está regulada (Eslava 2002, González et al. 2006). En tal sentido, con la finalidad de aportar información al conocimiento de la biología y dinámica poblacional de la especie, se consideró oportuno realizar la presente investigación con el objetivo de estimar los parámetros de crecimiento en longitud y peso de $H$. steindachneri, mediante el uso de los otolitos sagitales y frecuencia de tallas.

\section{MATERIALES Y MÉTODOS}

Muestreos mensuales de $H$. steindachneri fueron realizados desde julio 2005 hasta junio 2006, en la ranchería de la señora Ramona Marín ${ }^{\dagger}$, donde desembarca el $20 \%$ de la flota artesanal de Boca del Río, constituida por 69 embarcaciones operativas (González et al. 2006) que faenan en el área comprendida entre $10^{\circ} 50$ ' $38^{\prime \prime}-10^{\circ} 50^{\prime} 45^{\prime \prime} \mathrm{N}$ y los $64^{\circ} 07^{\prime} 29^{\prime \prime}-64^{\circ}$ 07 '41' W, del suroeste de la isla de Margarita (Fig. 1).

A cada individuo se le tomó la longitud total (L) con un ictiómetro marca Wilco, modelo 118, con una apreciación de $1 \mathrm{~mm}$; y el peso total (W), mediante el uso de una balanza electrónica, marca Sartorius, modelo 1106, con una apreciación de $0.01 \mathrm{~g}$ y $1000 \mathrm{~g}$ de capacidad máxima. La determinación del sexo fue establecida según lo sugerido por Holden \& Raitt (1975).

Posteriormente, fueron extraídos los otolitos del primer tercio del tabique ventral de la cavidad ótica, exponiendo el laberinto auditivo

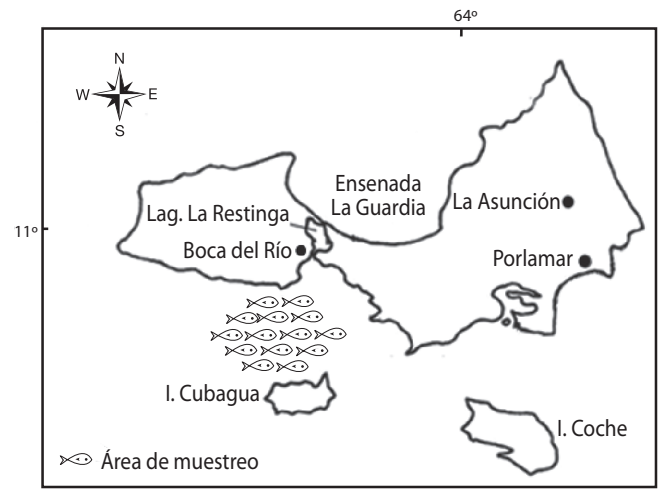

Fig. 1. Ubicación del área de pesca de la flota artesanal de Boca del Río, isla de Margarita, Venezuela.

Fig. 1. Location of the fishing area of the handcrafted fleet of Boca del Río, Margarita Island, Venezuela. 
para extraer con pinzas los otolitos sagitales y luego lavarlos con agua y jabón. Una vez secos, se guardaron en sobres de papel rotulados. La lectura de los anillos fue hecha con una lupa estereoscópica marca Wild, sumergidos en una cápsula de petri con glicerina y luz reflejada (Pascual 1996), determinándose el porcentaje de otolitos con anillos legibles y no legibles. La medición de los radios se realizó con una escala micrométrica de 100 divisiones adaptada a un ocular con objetivo de 16 X (González 1977, Morales 1991).

Los datos fueron procesados en dos hojas de Microsoft Office Excel Professional Edition 2003; en una fueron agrupados los individuos de la muestra por mes, longitud total, peso total y sexo; y en la otra, los datos de longitudes de los anillos de crecimiento en los otolitos sagitales, así como las longitudes de los peces, estimadas por retrocálculo (Tresierra \& Culquichicon 1993, Tresierra et al. 1995).

Se determinó la relación talla-peso mediante la ecuación propuesta por Ricker (1975): $\mathrm{W}=\mathrm{a} * \mathrm{~L}^{\mathrm{b}}$; donde $\mathrm{W}$ es el peso total del pez $(\mathrm{g})$ sin eviscerar, L es la longitud total ( $\mathrm{mm}), a$ es la intersección del eje de las ordenadas y $b$ es la pendiente de la curva. Las constantes $a$ y $b$ fueron obtenidas por el método de los mínimos cuadrados, previa linearización de los datos mediante una transformación logarítmica (Pauly 1983). Se determinó la existencia de diferencias significativas entre los coeficientes de machos y hembras ( $a$ y $b$ ) de la relación talla-peso, y se probó la hipótesis del crecimiento isométrico mediante una prueba $\mathrm{t}$ student con un nivel de significancia de $\alpha=0.05$, con el software Statgraphics Centurion XV.

Para la aplicación de la ecuación de retrocálculo, fue establecida la relación entre la longitud del pez (L) y el radio total del otolito (R), mediante una regresión lineal (González \& Mengual 1995), luego se estimaron las longitudes del pez en años previos de vida (retrocálculo) mediante la formula de R. Lee (Ricker 1975): $\mathrm{L}_{\mathrm{i}}=\mathrm{r}_{\mathrm{i}} / \mathrm{R}(\mathrm{L}-\mathrm{a})+\mathrm{a}$; donde $\mathrm{L}_{\mathrm{i}}$ es la longitud del pez en años previos de vida, $r_{j}$ el radio parcial del anillo, $\mathrm{R}$ el radio total del otolito, $\mathrm{L}$ la longitud del pez y "a", la intersección del eje de las ordenadas. Posteriormente se elaboró una clave edad-talla con las longitudes medias de $\mathrm{L}_{\mathrm{i}}$ (Sparre \& Venema 1997).

Con el propósito de validar los anillos como indicadores del crecimiento, fue utilizado el incremento marginal (IM) de la estructura dura seleccionada (González \& Mengual 1995, González \& Eslava 1999), a través de la relación: $\mathrm{IM}=\mathrm{Rn} / \mathrm{Rn}-1$; donde $\mathrm{Rn}$ es la distancia $(\mathrm{mm})$ desde el borde del último anillo hasta el borde de la estructura y $\mathrm{R}_{\mathrm{n}-1}$ la distancia (mm) desde el borde del penúltimo anillo hasta el borde de la estructura.

\section{Estimación de los parámetros de crecimiento:}

\section{a. Otolitos:}

Los parámetros de crecimiento, longitud asintótica o máxima teórica que el pez puede alcanzar $\left(\mathrm{L}_{\infty}\right)$ y el coeficiente de crecimiento $(\mathrm{k})$, se estimaron mediante el modelo numérico de Ford-Walford (Walford 1946), que correlaciona las longitudes de los individuos por grupos de edad, según la ecuación derivada de von Bertalanffy (Beverton \& Holt 1957): $\mathrm{L}_{\mathrm{t}+1}=\mathrm{L}_{\infty}\left(1-\mathrm{e}^{-}\right.$ $\left.{ }^{\mathrm{k}}\right)+\mathrm{L}_{\mathrm{t}}{ }^{*} \mathrm{e}^{-\mathrm{k}}$. El modelo establece que el punto en que la bisectriz intercepta la recta de regresión es igual a $\mathrm{L}_{\infty}$, por lo tanto, la fórmula pasa a ser representada por la ecuación de regresión lineal (Pinto et al. 2004), útil para estimar posteriormente los valores $\mathrm{L}_{\infty} \mathrm{y} \mathrm{k}$ a través del las constantes de regresión $a$ y $b$.

\section{b. Frecuencia de tallas}

Se hizo uso del software FiSAT, el cual se basa en el análisis de frecuencia de longitudes por mes (Gayanilo et al. 1996), para estimar los parámetros de crecimiento con la rutina ELEFAN I, que emplea el análisis de la progresión modal, previa descomposición de la frecuencia de longitudes según el método de Bhattacharya (1967) y optimización de las estimaciones de $\mathrm{L}_{\infty}$ y k mediante el modelo de Powell (1979) y Wetherall (1986). Posteriormente se 
calculó el índice de crecimiento phi prima (Ø') mediante la ecuación de Munro \& Pauly (1983): $\varnothing^{\prime}=\log _{10} \mathrm{k}+2 \log _{10} \mathrm{~L}_{\infty}, \mathrm{y}$ poder comparar los parámetros de crecimiento obtenidos por los métodos directo e indirecto, mediante el coeficiente de variación (CV) del Ø’' (Pauly 1983, Sparre \& Venema 1997).

Para la estimación de la curva de crecimiento del modelo de von Bertalanffy fue estimado el $\mathrm{t}_{0}$ con la aplicación de la fórmula de Pauly (1979): $\log _{10}\left(-t_{0}\right)=-0.3922-0.2752$ $\log _{10} \mathrm{~L}_{\infty}-1.038 \log _{10} \mathrm{k}$; donde $\mathrm{t}_{0}$ es la constante que representa la edad que supuestamente debe tener el pez para que su longitud o peso sea igual a cero; y la edad límite mediante la ecuación de Taylor (1958): $\mathrm{A}_{0.95}=\mathrm{t}_{0}+2.996 / \mathrm{k}$; donde $\mathrm{A}_{0.95}$ es la edad límite o tiempo requerido para alcanzar el $95 \%$ de la longitud máxima $\left(\mathrm{L}_{\infty}\right)$.

\section{c. Curva de crecimiento}

Por otro lado, se estimaron las curvas de crecimiento en longitud y en peso de $H$. steindachneri, fueron obtenidas con la ecuación de von Bertalanffy (Beverton \& Holt 1957) y la edad límite calculada: $\mathrm{L}_{\mathrm{t}}=\mathrm{L}_{\infty}\left[1-\mathrm{e}^{-\mathrm{k}(\mathrm{t}-\mathrm{to})}\right] ; \mathrm{W}_{\mathrm{t}}=\mathrm{W}_{\infty}\left[1-\mathrm{e}^{-\mathrm{k}(\mathrm{t}-\mathrm{to})}\right]^{\mathrm{b}} . \mathrm{El}$ peso asintótico fue determinado por la relación talla-peso, mediante el modelo: $\mathrm{W}_{\infty}=$ $\mathrm{a} * \mathrm{~L}_{\infty}^{\mathrm{b}}$.

\section{RESULTADOS}

De los 953 ejemplares examinados, 540 (56.7\%) fueron machos y 413 (43.3\%) hembras, con longitudes comprendidas entre 103 y $226 \mathrm{~mm}$, y pesos que oscilaron entre 11.83 y 192.31g (Cuadro 1). Respecto a las tallas, las hembras presentaron tallas ligeramente más grandes que los machos, pero al no encontrarse diferencias significativas $\left(t_{\mathrm{s}}=0.5367 ; p>0.05\right)$,

CUADRO 1

Ejemplares examinados del pez, Haemulon steindachneri, del suroeste de la isla de Margarita, Venezuela, durante el periodo julio 2005 - junio 2006

TABLE 1

Specimens examined of the grunt, Haemulon steindachneri, from southwest Margarita Island, Venezuela, between July 2005 and June 2006

\begin{tabular}{|c|c|c|c|c|c|c|c|c|c|c|}
\hline \multirow{3}{*}{$\begin{array}{c}\text { Mes / } \\
\text { Año }\end{array}$} & \multicolumn{5}{|c|}{ MACHOS } & \multicolumn{5}{|c|}{ HEMBRAS } \\
\hline & \multirow{2}{*}{$\mathrm{n}$} & \multicolumn{2}{|c|}{ Longitud total (mm) } & \multicolumn{2}{|c|}{ Peso total (g) } & \multirow{2}{*}{$\mathrm{n}$} & \multicolumn{2}{|c|}{ Longitud total $(\mathrm{mm})$} & \multicolumn{2}{|c|}{ Peso total (g) } \\
\hline & & Min - Max & Media & Min - Max & Media & & Min - Max & Media & Min - Max & Media \\
\hline jul-05 & 76 & $127-194$ & 163 & $28.57-109.22$ & 70.59 & 56 & $122-206$ & 164 & $29.8-163.99$ & 72.36 \\
\hline ago-05 & 33 & $149-176$ & 162 & $45.69-77.82$ & 62.57 & 37 & $141-188$ & 162 & $38.77-95.51$ & 62.61 \\
\hline sep-05 & 37 & $144-169$ & 158 & $46.15-75.46$ & 60.25 & 12 & $142-184$ & 158 & $50.72-97.99$ & 60.1 \\
\hline oct- 05 & 43 & $138-217$ & 154 & $47.63-168.82$ & 63.99 & 30 & $136-179$ & 154 & $44.17-94.86$ & 64.46 \\
\hline nov-05 & 32 & $122-213$ & 168 & $28.39-171.06$ & 82.77 & 38 & $142-211$ & 169 & $46.63-192.31$ & 83.48 \\
\hline dic-05 & 45 & $118-198$ & 142 & $26.66-109.22$ & 46.56 & 25 & $123-154$ & 141 & $30.14-61.36$ & 46.06 \\
\hline ene-06 & 51 & $103-203$ & 172 & $11.83-127.5$ & 79.44 & 45 & $140-187$ & 171 & $42.36-117.42$ & 78.91 \\
\hline feb-06 & 42 & $142-195$ & 168 & $21.92-117.18$ & 64.47 & 40 & $147-205$ & 169 & $26.58-118.88$ & 66.54 \\
\hline mar-06 & 31 & $147-179$ & 161 & $24.38-92.52$ & 64.94 & 42 & $149-185$ & 161 & $34.32-98.83$ & 64.37 \\
\hline abr-06 & 49 & $162-226$ & 188 & $72.04-159.24$ & 108 & 27 & $165-226$ & 188 & $68.65-141.14$ & 108.91 \\
\hline may-06 & 48 & $146-203$ & 177 & $44.25-173.72$ & 90.84 & 32 & $152-201$ & 177 & $52.3-131.19$ & 90.66 \\
\hline jun-06 & 53 & $142-199$ & 177 & $44.62-129.05$ & 90.69 & 29 & $154-208$ & 177 & $61.65-137.33$ & 90.81 \\
\hline TOTAL & 540 & & & & & 413 & & & & \\
\hline
\end{tabular}

* $\mathrm{n}=$ número de ejemplares examinados; Min = valor mínimo; Max = valor máximo. $\mathrm{n}=$ number of specimens examined; Min = minimum value; Max $=$ maximum value 
se trataron los sexos combinados para la determinación de la edad y el crecimiento.

Determinación de los grupos de edad: La población de estudio estuvo constituida por 4 grupos de edad: $1^{+}(10.8 \%), 2^{+}(30.1 \%), 3^{+}$ $(44.5 \%)$ y $4^{+}(14.6 \%)$, representados por igual número de anillos hialinos (Fig. 2); con longitudes comprendidas entre 39 y $107 \mathrm{~mm}$ (Grupo $1^{+}$), entre 65 y $149 \mathrm{~mm}\left(\right.$ Grupo $2^{+}$), entre 83 y $182 \mathrm{~mm}\left(\right.$ Grupo $3^{+}$), y entre 113 y $189 \mathrm{~mm}$ (Grupo 4+) (Fig. 3).

Relación talla-peso: La relación tallapeso (Fig. 4) no presentó diferencias significativas en las intercepciones $a \quad\left(\mathrm{t}_{\mathrm{s}}=-1.17\right.$;
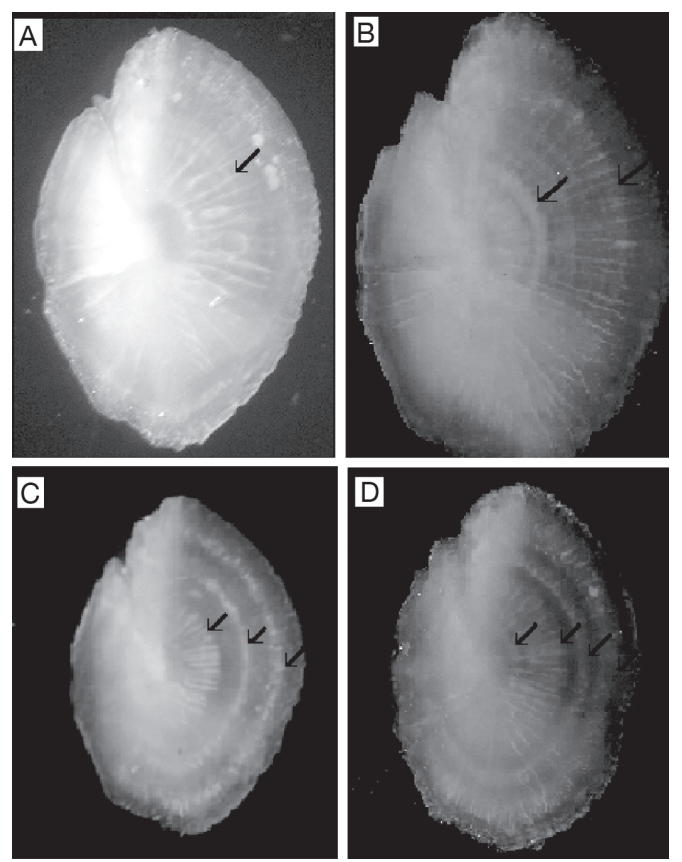

Fig. 2. Otolitos del pez, Haemulon steindachneri. (A) Grupo de edad $1^{+}$: L=148 mm. Objetivo 16 X. (B) Grupo de edad $2^{+}: \mathrm{L}=184 \mathrm{~mm}$. Objetivo $16 \mathrm{X}$. (C) Grupo de edad $3^{+}: \mathrm{L}=204 \mathrm{~mm}$. Objetivo $6.4 \mathrm{X}$. (D) Grupo de edad $4^{+}$: $\mathrm{L}=216 \mathrm{~mm}$. Objetivo $6.4 \mathrm{X}$.

Fig. 2. Otoliths of the grunt, Haemulon steindachneri. (A) Group of age $1^{+}: \mathrm{L}=148 \mathrm{~mm}$. Objective 16 X. (B) Group of age $2^{+}: \mathrm{L}=184 \mathrm{~mm}$. Objective $16 \mathrm{X}$. (C) Group of age $3^{+}$: $\mathrm{L}=204 \mathrm{~mm}$. Objective 6.4 X. (D) Group of age $4^{+}: \mathrm{L}=216$ $\mathrm{mm}$. Objective $6.4 \mathrm{X}$. $p>0.05)$ y pendientes $b\left(\mathrm{t}_{\mathrm{S}}=-1.81 ; p>0.05\right)$ de hembras y machos. Además, el parámetro $b$ de esta relación fue significativamente diferente de $3\left(\mathrm{t}_{\mathrm{s}}=3.5186 ; p<0.05\right)$; de tal manera que dicha relación quedó establecida para ambos sexos en: $\mathrm{W}=0.0003 * \mathrm{~L}^{2.89}$, con un crecimiento alométrico.

\section{Parámetros y curvas de crecimiento:} La relación entre la longitud total del pez y el radio total del otolito, respondió al modelo lineal: $\mathrm{L}=1.13+46.77^{*} \mathrm{R}$, con un elevado coeficiente de correlación de Pearson: $\mathrm{r}=0.90$ (Fig. 5). Posteriormente se retrocalcularon las longitudes por cada grupo de edad (Cuadro 2). Las medias de estas longitudes se utilizaron para estimar los parámetros de crecimiento al aplicar el modelo numérico de Ford-Walford (Walford 1946), obteniéndose: $\mathrm{L}_{\infty}=231 \mathrm{~mm}$;

\section{CUADRO 2}

Clave edad-talla del pez, Haemulon steindachneri, del suroeste de la isla de Margarita, Venezuela

TABLE 2

Age-length key for the grunt, Haemulon steindachneri, from southwest Margarita Island, Venezuela

\begin{tabular}{ccccc} 
TALLA & \multicolumn{4}{c}{ EDAD } \\
$(\mathrm{mm})$ & $1^{+}$ & $2^{+}$ & $3^{+}$ & $4^{+}$ \\
$30-39$ & 2 & - & - & - \\
$40-49$ & 60 & - & - & - \\
$50-59$ & 231 & - & - & - \\
$60-69$ & 329 & 1 & - & - \\
$70-79$ & 229 & 45 & - & - \\
$80-89$ & 32 & 203 & 1 & - \\
$90-99$ & 4 & 251 & 12 & - \\
$100-109$ & 2 & 152 & 62 & - \\
$110-119$ & - & 80 & 135 & 2 \\
$120-129$ & - & 34 & 146 & 8 \\
$130-139$ & - & 23 & 94 & 34 \\
$140-149$ & - & 4 & 37 & 42 \\
$150-159$ & - & - & 31 & 20 \\
$160-169$ & - & - & 6 & 14 \\
$170-179$ & - & - & 1 & 7 \\
$180-189$ & - & - & 1 & 3 \\
Media & 64.59 & 97.83 & 124.67 & 146.66 \\
Desviación & \pm 9.78 & \pm 13.94 & \pm 14.61 & \pm 14.39 \\
típica & & & & \\
& & & &
\end{tabular}




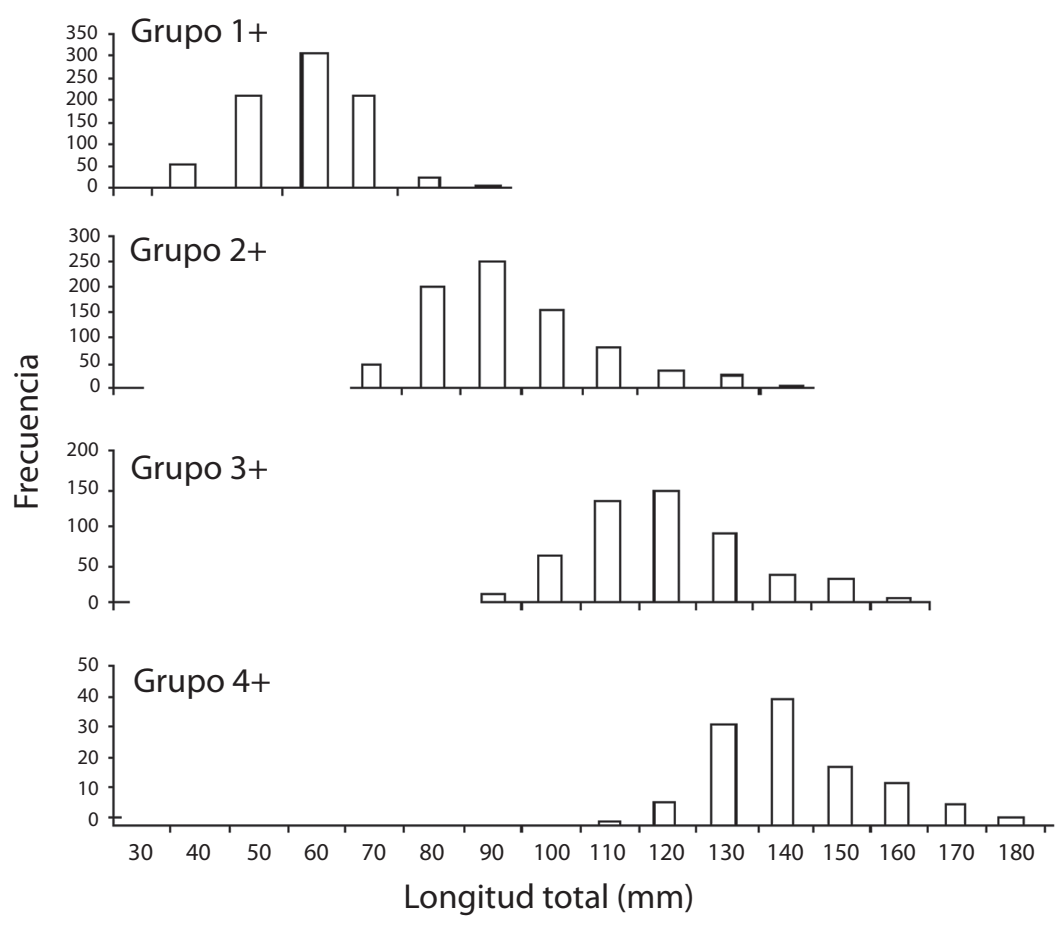

Fig. 3. Estructura de tallas por grupo de edad, mediante el análisis de otolitos del pez, Haemulon steindachneri.

Fig. 3. Length frequency of the groups of age by the otoliths analysis of the grunt, Haemulon steindachneri.

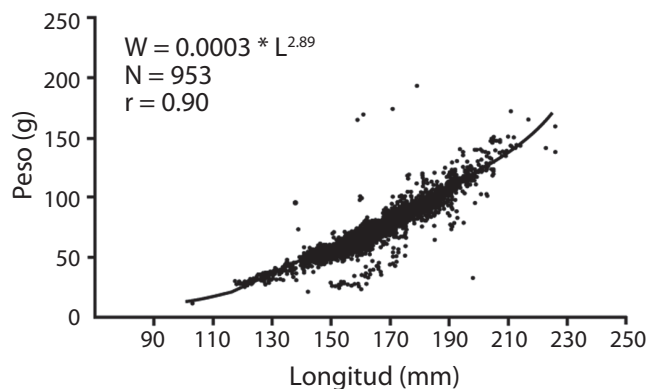

Fig. 4. Relación talla-peso del pez, Haemulon steindachneri, del suroeste de la isla de Margarita, Venezuela, durante el periodo julio 2005-junio 2006.

Fig. 4. Length-weigh relation, of the grunt, Haemulon steindachneri, from southwest Margarita Island, Venezuela, between July 2005 and June 2006.

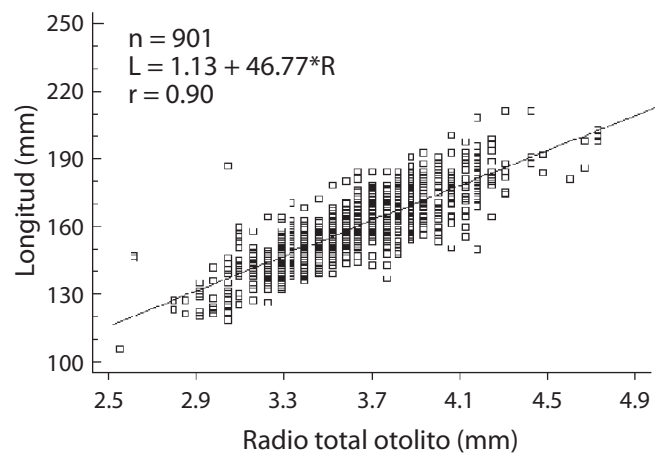

Fig. 5. Relación longitud del pez y radio del otolito del pez, Haemulon steindachneri.

Fig. 5. Total length-otolith radio relation of the grunt, Haemulon steindachneri. 
$\mathrm{k}=0.569 \mathrm{año}^{-1}$. En la evolución mensual del incremento marginal (IM) de los otolitos (Fig. 6) se pueden apreciar las medias mensuales. El menor valor correspondió al mes de abril, lo que sugiere que el anillo hialino se forma en este mes. Igualmente se calcularon los parámetros de crecimiento mediante el análisis de frecuencia de tallas, con el modelo de Powell (1979) y Wetherall (1986) (Fig. 7), la longitud asintótica estimada fue: $\mathrm{L}_{\infty}=228 \mathrm{~mm}$, $\mathrm{y}$ el coeficiente de crecimiento: $\mathrm{k}=0.580 \mathrm{año}^{-1}$. Posteriormente fue obtenido el peso asintótico $\left(\mathrm{W}_{\infty}\right)$ al considerar las constantes $a$ y $b$ de la relación talla-peso y la longitud asintótica $\left(\mathrm{L}_{\infty}\right)$, obteniéndose: $\mathrm{W}_{\infty}=203 \mathrm{~g}$ para el método directo y $\mathrm{W}_{\infty}=195 \mathrm{~g}$ para el método indirecto. Los parámetros de crecimiento $\left(\mathrm{L}_{\infty} \mathrm{y} \mathrm{k}\right)$ obtenidos por ambos métodos con su coeficiente de variación (CV) del phi prima (Ø'), son presentados en el Cuadro 3. Finalmente se presentan las curvas de crecimiento en longitud y peso según el método directo e indirecto, ajustadas al modelo de von Bertalanffy (Beverton \& Holt 1957) (Fig. 8) con el $t_{0}$ promedio y la edad límite estimados para ambos métodos en: $\mathrm{t}_{\mathrm{o}}=-$ 0.8 años y $\mathrm{A}_{0.95}=4$ años.

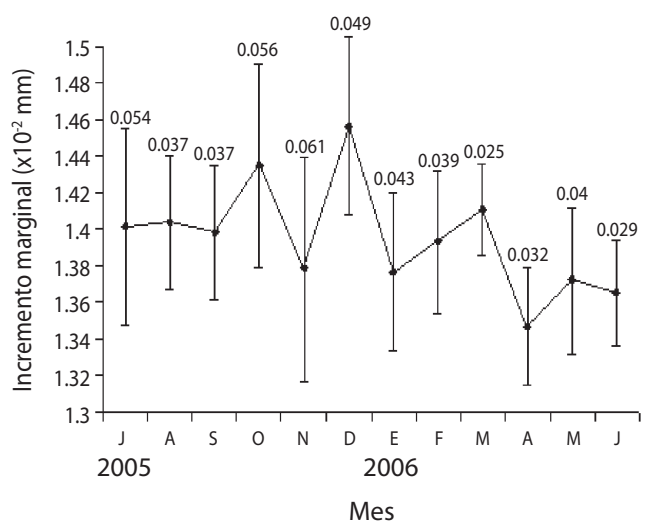

Fig. 6. Promedio mensual de los incrementos marginales en otolitos del pez, Haemulon steindachneri.

Fig. 6. Otoliths marginal increases monthly average from the grunt, Haemulon steindachneri.

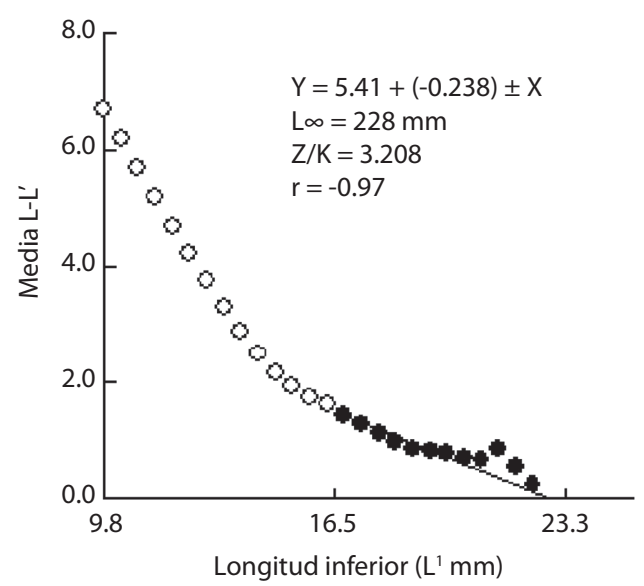

Fig. 7. Estimación de $\mathrm{L}_{\infty}$, aplicando el modelo de Powell (1979) y Wetherall (1986) del pez, Haemulon steindachneri, del suroeste de la isla de Margarita, Venezuela, durante el periodo julio 2005-junio 2006.

Fig. 7. Estimation of $\mathrm{L}_{\infty}$, applying the Powell (1979) and Wetherall (1986) model of the grunt, Haemulon steindachneri, from southwest Margarita Island, Venezuela, between July 2005 and June 2006.

\section{DISCUSIÓN}

El recuento de los anillos de crecimiento en otolitos, permitió establecer cuatro grupos de edad $\left(1^{+}, 2^{+}, 3^{+}, 4^{+}\right)$; resultado que contrasta con Eslava (1991), quien determinó tres grupos de edad $\left(1^{+}, 2^{+}, 3^{+}\right)$por medio del cleitrum. Esta diferencia, posiblemente se deba a que los otolitos son mejores estructuras para determinar la edad por su mejor legibilidad de los anillos de crecimiento en $H$. steindachneri (Morales 2007). Es importante acotar que no se obtuvieron ejemplares pertenecientes al grupo de edad $0^{+}$, por no encontrarse en las muestras provenientes de las capturas comerciales de la flota.

Las medias de los incrementos marginales (IM) mensuales mostraron el valor mínimo en el mes de abril. La formación de los anillos de crecimiento en este pez, posiblemente está relacionada con las variaciones del metabolismo causadas por el desarrollo gonadal y los gastos de energía producto del desove, debido a que el menor valor IM coincide con la época de reproducción, pauta observada por Rodríguez 
CUADRO 3

Validación de los parámetros de crecimiento, a través del coeficiente de variación (CV) del índice de crecimiento phi prima (Ø’) del pez, Haemulon steindachneri, del suroeste de la isla de Margarita, Venezuela

TABLE 3

Validation of growth parameters, applying the variation coefficient (CV) of the growth rate phi prime (Ø') of the grunt, Haemulon steindachneri, from southwest Margarita Island, Venezuela

\begin{tabular}{lccc}
\multicolumn{1}{c}{ ESTADÍSTICO } & $\mathrm{L}_{\infty}(\mathrm{mm})$ & $\mathrm{k}\left(\mathrm{año}^{-1}\right)$ & $\varnothing^{\prime}$ \\
Método directo (otolitos) & 231 & 0.569 & 4.482 \\
Método indirecto (frecuencia de tallas) & 228 & 0.580 & 4.479 \\
Media & 230 & 0.575 & 4.481 \\
Desviación típica & \pm 2.12 & \pm 0.008 & \pm 0.002 \\
Coeficiente de variación & - & - & $0.04 \%$
\end{tabular}
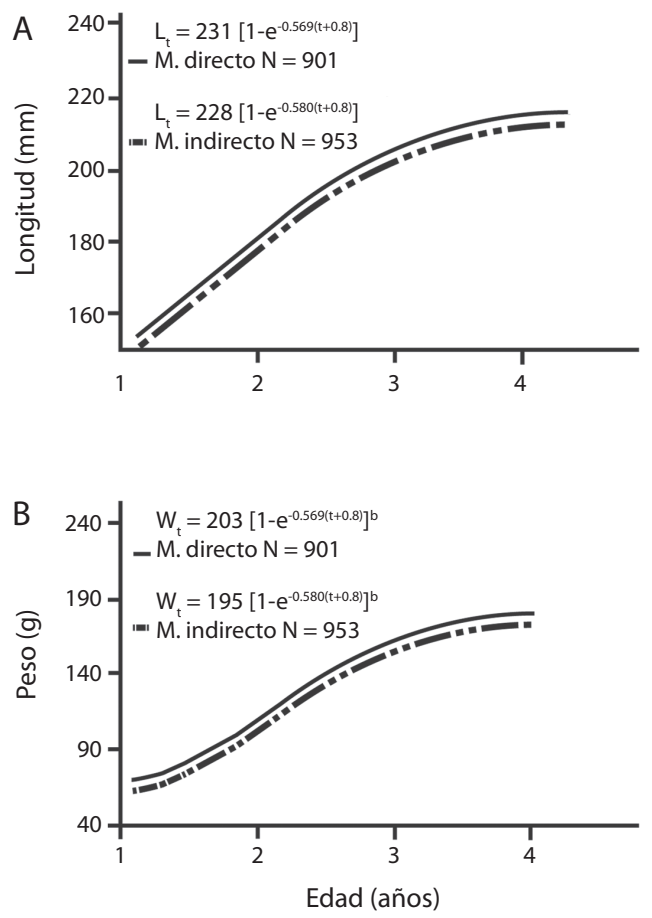

Fig. 8. Curvas de crecimiento en longitud (A) y peso (B), obtenidos por los métodos directo e indirecto, según el modelo de von Bertalanffy del pez, Haemulon steindachneri, del suroeste de la isla de Margarita, Venezuela, durante el periodo julio 2005-junio 2006.

Fig. 8. Length (A) and weight (B) growth curves obtained by direct and indirect methods, according to the von Bertalanffy model, of the grunt, Haemulon steindachneri, from southwest Margarita Island, Venezuela, between July 2005 and June 2006.
(1985) en H. steindachneri del oriente del país, y García (1992) en Haemulon album, H. plumieri y H. sciurus de las costas de Cuba; se indica, además, que el periodo reproductor de las hembras maduras ocurre de marzo a julio, con un periodo de desove que se prolonga hasta noviembre; sugiriendo la formación de un anillo hialino anual alrededor del mes de abril. Por otra parte, las causas de formación de los anillos parece guardar cierta relación con el régimen de lluvias, responsable del suministro de alimentos por descarga de los grandes ríos suramericanos, como son el Amazonas, el Orinoco y el Esequibo, que influyen en las costas de la región oriental de Venezuela, a través de las corrientes horizontales (González et al. 1998).

Según los datos históricos del Servicio de Meteorología de la Fuerza Aérea Venezolana (1990-2006), en el mes de abril 2006 la precipitación fue baja y la velocidad del viento alta, lo que indica una fuerte surgencia que produce una disminución de la temperatura del agua; esta condición podría tener un efecto en la tasa de crecimiento y época de reproducción de la especie. Por otra parte, Tolentino \& Mendoza (2001) destacan que las desviaciones observadas en el IM, pueden ser consecuencia de la variabilidad intrínseca de la población.

En la relación longitud total del pez-radio total del otolito, se puede apreciar que las longitudes retrocalculadas fueron mayores en los 
ejemplares de más edad y la línea de regresión no pasó por el origen de las coordenadas. Esto unido al valor alto del coeficiente de correlación obtenido, satisface la premisa básica de isometría que debe existir entre las tasas de crecimiento del pez y alguna de sus estructuras duras (Aguayo 1974, Pinto et al. 2004), lo que indica un crecimiento alométrico lineal del otolito, probablemente por la presencia del fenómeno de Lee (1920).

El coeficiente de regresión $b=2.89$ de la relación talla-peso, indicó un crecimiento alométrico; esta condición biológica muestra un acelerado crecimiento en longitud, el cual puede variar entre las especies y estar relacionado con el tamaño de la muestra, época del año, alimentación, estado de madurez, factores ambientales y con el rápido crecimiento de las primeras etapas de vida (Tresierra \& Culquichicon 1993, Gómez et al. 2002). El valor coincide con $b=2.62$ y $b=2.87$ obtenidos por Rodríguez (1985) y Barreto (1996), respectivamente. Otros autores señalan valores muy similares en Haemulon aurolineatum (Billing \& Munro 1974, Kossowski 1985, Parra 1996) y Ortopristis ruber (Guzmán 1990).

El ejemplar que presentó la mayor talla en todo el año de muestreo midió $226 \mathrm{~mm}$, valor similar a las longitudes asintóticas obtenidas por los métodos directo $\left(\mathrm{L}_{\infty}=231 \mathrm{~mm}\right)$ e indirecto $\left(\mathrm{L}_{\infty}=228 \mathrm{~mm}\right)$. Sin embargo, están por debajo de lo señalado por Cervigón (1993) y González et al. (2006) quienes reportaron tallas de $263 \mathrm{~mm}$. Estas diferencias sugieren la necesidad de establecer medidas de manejo de la especie y de las pesquerías multiespecíficas de la región, para evitar la sobreexplotación del recurso, más aún cuando la especie no posee ningún tipo de regulación pesquera (González et al. 2006).

Los coeficientes de crecimiento determinados para los métodos directo $\left(\mathrm{k}=0.569 \mathrm{año}^{-1}\right)$ e indirecto $\left(\mathrm{k}=0.580 \mathrm{año}^{-1}\right)$, indican que la especie posee un crecimiento moderadamente rápido, coincidiendo con lo señalado por Barreto (1996) en el oriente venezolano. Dicho patrón de crecimiento puede tener relación con la presión por parte de los depredadores y con factores intrínsecos de la especie (Carpenter 2002), adaptándose los valores de $\mathrm{k}$ al rango señalado por Pauly (1979) para peces tropicales. A su vez, Gulland (1971), señaló que el coeficiente de crecimiento $(\mathrm{k})$, de la ecuación de von Bertalanffy, guarda cierta proporcionalidad con la mortalidad natural de la población, es decir, un pez que alcanza la longitud asintótica rápido ( $\mathrm{k}$ elevado), es probable que tenga una mortalidad natural elevada, mientras que un pez que crece lentamente ( $\mathrm{k}$ bajo), es posible que tenga una baja mortalidad natural.

En la curva de crecimiento en longitud y peso, se evidenció un crecimiento rápido en los dos primeros años de vida, el cual se hace más lento después del segundo año, hasta que alcanzan su longitud máxima, cerca del cuarto año de vida, donde la pendiente se hace prácticamente asintótica. Se observó que la curva de crecimiento en peso se comporta de manera sigmoidea, esto tiene que ver con el hecho de que en las primeras etapas de vida, el pez utiliza parte de la energía para el desarrollo gonadal (Ricker 1975, Csirke 1980). Por otra parte, Gómez-Larrañeta (1967), indicó que la temperatura y la abundancia de alimento, son factores ambientales importantes que influyen sobre la curva de crecimiento, es decir, una especie puede tener sus propios parámetros medios de crecimiento, pero éstos pueden variar por las características del área donde se desarrolla la población.

El valor bajo del coeficiente de variación del índice de crecimiento phi prima ( $\left.\varnothing^{\prime}\right)$ no excedió el $4 \%$, lo cual se asume que los resultados son estadísticamente similares (Pauly 1983, Sparre \& Venema 1997). Esta similitud indica que los parámetros de crecimiento de H. steindachneri, obtenidos con los métodos directo e indirecto, son válidos en su aplicación (Munro \& Pauly 1983). Por tal razón, el presente estudio sugiere que ambos métodos son adecuados para la determinación de la edad y el crecimiento de H. steindachneri. Sin embargo, se sugiere la aplicación del método indirecto porque proporciona los mismos resultados con mayor facilidad en el trabajo de laboratorio. 


\section{AGRADECIMIENTOS}

Expresamos nuestro más sincero agradecimiento a Ramona Marín ${ }^{\dagger}$ y a su esposo Jesús Marcano por su apoyo logístico en la obtención de las muestras biológicas. A Nora Eslava en el análisis y discusión de los datos. A Francisco Guevara por su ayuda en el laboratorio de Biología Pesquera del IIC-UDONE.

\section{RESUMEN}

La edad y el crecimiento del pez Haemulon steindachneri fueron determinados por medio de los otolitos sagitales (método directo) y la distribución de frecuencia de tallas (método indirecto). Un total de 953 individuos fueron capturados por la flota artesanal de Boca del Río, suroeste de la isla de Margarita, entre julio 2005-junio 2006. Se estableció la relación talla-peso y se estimaron los parámetros de crecimiento de las curvas en longitud y peso del modelo de von Bertalanffy. El recuento de los anillos anuales en otolitos, permitió establecer cuatro grupos de edad, siendo el grupo $3^{+}$el más representativo, con un $44.5 \%$ de la población muestreada. El cálculo de los incrementos marginales del crecimiento mensual de los otolitos, sugirió la formación de un anillo hialino anual en el mes de abril, el cual coincide con la época máxima de reproducción. No se observó diferencia significativa en las pendientes $b\left(\mathrm{t}_{\mathrm{s}}=-\right.$ $1.81 ; p>0.05)$ e interceptos $a\left(\mathrm{t}_{\mathrm{s}}=-1.17 ; p>0.05\right)$ de hembras y machos, con una relación talla-peso para ambos sexos de $\mathrm{W}=0.0003 * \mathrm{~L}^{2.89}$, la que indicó un crecimiento alométrico. Los parámetros de crecimiento quedaron establecidos en: $\mathrm{L}_{\infty}=231 \mathrm{~mm}, \mathrm{~W}_{\infty}=203 \mathrm{~g}, \mathrm{k}=0.569 \mathrm{año}^{-1}$ (método directo) y $\mathrm{L}_{\infty}=228 \mathrm{~mm}, \mathrm{~W}_{\infty}=195 \mathrm{~g}, \mathrm{k}=0.580 \mathrm{año}^{-1}$ (método indirecto), lo que sugiere que se trata de una especie de vida corta y crecimiento moderadamente rápido. El coeficiente de variación $(\mathrm{CV})$ del índice de crecimiento phi prima ( $\left.\varnothing^{\prime}\right)$, demostró semejanza de los parámetros de crecimiento en longitud obtenidos por los métodos directo e indirecto; por lo que se recomienda la aplicación del método indirecto, así como el análisis periódico de los parámetros poblacionales.

Palabras clave: edad, crecimiento, Haemulon steindachneri, isla Margarita, Venezuela.

\section{REFERENCIAS}

Aguayo, M. 1974. Estudio de la edad y crecimiento de la merluza de cola (Macruronus magellanicus, Lonnberg, 1907). Inv. Pesq. 19:1-43.
Araya, M. \& L. Cubillos. 2002. The retrospective analysis of the growth in fish and their associated problems. Gayana (Concepción) 66: 161-179.

Barreto, C. 1996. Edad y crecimiento de Haemulon steindachneri, capturados en la costa norte del estado Sucre, Venezuela. Tesis de Grado, Licenciatura en Biología, Universidad de Oriente, Venezuela.

Beverton, R.J.H \& S.J. Holt. 1957. On the dynamics of exploited fish populations, Fishery Investigations. Minist. Agric. Fish. Food G.B. (2 Sea Fish) 19: $1-533$.

Bhattacharya, C. 1967. A simple method of resolution of a distribution into Gaussian components. Biometrics 23: $115-123$.

Billing, V. \& J. Munro. 1974. The biology, ecology, exploitation and management of Caribbean reef fishes. Part. VI: The biology, ecology and bionomics of Caribbean reef fishes: Haemulidae (grunts). Zoology Department Research Report 3: 128.

Carpenter, K. 2002. The living marine resources of the western central Atlantic. Volume 3: Bony fishes part 2 (Opistognathidae to Molidae), sea turtles and marine mammals. FAO Species identification guide for fishery purposes and American Society of Ichthyologists and Herpetologists Special Publication 5: 1375-2127.

Cervigón, F. 1993. Los peces marinos de Venezuela Vol. IV. Ex Libris, Caracas, Venezuela.

Csirke, J. 1980. Introducción a la dinámica de poblaciones de peces. FAO Documento Técnico de Pesca No. 192. Roma, Italia.

Eslava, N. 1991. Comparación del uso de escamas y cleitra para estudios de edad y crecimiento del chere-chere, Haemulon steindachneri (Jordan y Gilbert 1882) (Teleostei: Haemulidae). Memoria de la Sociedad de Ciencias Naturales La Salle. 135-136: 97-107.

Eslava, N. 2002. Caracterización de las unidades de pesca artesanal del estado Nueva Esparta, Venezuela. Trabajo de Ascenso, Instituto de Investigaciones Científicas, Universidad de Oriente, Núcleo Nueva Esparta, Venezuela.

García, J. 1992. Edad y crecimiento de algunos peces neríticos de Cuba. Tesis Ph.D., Instituto de Oceanología, Academia de Ciencias de Cuba, Cuba.

Gayanilo, F., P. Sparre \& D. Pauly. 1996. Stock assessment tools users manual. FAO, Roma, Italia. 
Gómez-Larrañeta, M. 1967. Crecimiento de Pagellus erithrinus de las costas de Castellón. Inv. Pesq. 31: 185-258.

Gómez, G., R. Guzmán \& R. Chacón. 2002. Algunos aspectos de la biología reproductiva y poblacional del torroto, Genyatremus luteus, (Bloch 1797) (Pisces: Haemulidae) en el Golfo de Paria, Venezuela. Zoot. Trop. 20: 223-234.

González, L.W. 1977. Aspectos técnicos de preparación de otolitos para estudios de edad de algunas especies del género Cynoscion (Pisces: Sciaenidae). Lagena: 39-40: 43-48.

González, L.W. 1979. Determinación de la edad del Paralonchurus peruanus (Steindachner, 1875) (Pisces: Sciaenidae) mediante el uso de los otolitos. Informe Museo del Mar 22: 1-19.

González, L.W. 1985. Determinación de edad y crecimiento de la sardina Sardinella aurita (Valenciennes 1847) (Pisces: Clupeidae) de la región nor-oriental de Venezuela. Bol. Inst. Oceanogr. Venezuela 24: 111-128.

González, L.W. \& A. Mengual. 1995. Edad y crecimiento del machuelo Opisthonema oglinum (Le Sueur 1818) (Teleostei: Clupeidae) de la Isla de Margarita, Venezuela. Ciencias Marinas 21: 387-399.

González, L.W., N. Eslava \& C. Silva. 1998. Edad, crecimiento y mortalidad de Lutjanus purpureus Poey, 1867 (Pisces: Lutjanidae) de la región de Guayanas. Bol. Invest. Mar. Cost. 27: 7-20.

González, L.W. \& N. Eslava. 1999. Edad y crecimiento del pargo colorado Lutjanus purpureus Poey, 1867 (Teleostei: Lutjanidae) de la región oriental de Venezuela. Rev. Biol. Mar. Oceanogr. 34: 99-107.

González, L.W., N. Eslava \& F. Guevara. 2006. Catálogo de la pesca artesanal del estado Nueva Esparta, Venezuela. Radoca, Cumaná, Venezuela.

Gulland, J. 1971. Manual de métodos para la evaluación de poblaciones de peces. Acribia, Zaragoza, España.

Guzmán, R. 1990. Aspectos biológicos y pesqueros del corocoro, Orthopristis ruber (Cuvier 1830) (Pisces: Haemulidae), en áreas de la Isla de Margarita y Costa Norte del Edo. Sucre, Venezuela. Tesis de Maestría, Instituto Oceanográfico de Venezuela, Cumaná, Venezuela.

Heald, E.J \& R. Griffiths. 1967. The age determination, from scale reading of the sardine, Sardinella anchovia of the Gulf of Cariaco, Eastern Venezuela. Ser. Rec. Explot. Pesq. (MAC) 1: 375-446.
Holden, M.J. \& D.F.S. Raitt. 1975. Manual de ciencia pesquera. Parte 2. Métodos para investigar los recursos y su aplicación. FAO Documento Técnico de Pesca No. 115. Roma, Italia.

Humann, P. \& N. Deloach. 2002. Reef fish identification. Florida, Caribbean, Bahamas. New World, Jacksonville, Florida.

Kossowski, A. 1985. Reproducción del cují, Haemulon aurolineatum (Cuvier 1829) en la Isla de Margarita. Tesis de Grado, Licenciatura en Biología, Mención Biología Marina, Universidad de Oriente, Venezuela.

Lee, R. 1920. A review of the methods of age and growth determination in fishes by means of scales. Ministry of Agriculture and Fishery Investigations 4: 19-28.

Morales, B. 1991. Determinación del crecimiento de peces óseos en base a la microestructura de los otolitos. FAO Documento Técnico de Pesca No. 322. Roma, Italia.

Morales, M. 2007. Determinación de edad y crecimiento del chere-chere, Haemulon steindachneri (Jordan y Gilbert, 1882) (Teleostei: Haemulidae) del suroeste de la Isla de Margarita. Tesis de Grado, Licenciatura en Biología Marina, Universidad de Oriente, Venezuela.

Munro, J. \& D. Pauly. 1983. A simple method for comparing the growth of fishes and invertebrates. Fishbyte $1: 5-6$.

Palazón, J. L. \& L. W. González. 1986. Edad y crecimiento del pargo cebal, Lutjanus analis (Cuvier 1828) (Teleostei: Lutjanidae) en la Isla de Margarita y alrededores, Venezuela. Inv. Pesq. 50: 151-165.

Parra, B. 1996. Relación longitud-peso, factor de condición e índice hepático de Haemulon aurolineatun (Cuvier 1830) (Pisces: Haemulidae) de la Isla de Cubagua, Venezuela. Acta Cient. Venez. 47: 122-134.

Pascual, A. 1996. Métodos para el estudio de la edad y crecimiento en peces. Programa de Pesca VECEP ALA 92/43. Instituto Nacional de Pesca, Guayaquil, Ecuador.

Pauly, D. 1979. Theory and management of tropical multispecies stocks: a review, with emphasis on the Southeast Asian demersal fisheries. International Center for Living Aquatic Resources Management. Studies and Review 1: 1-35.

Pauly, D. 1983. Algunos métodos simples para la evaluación de recursos pesqueros tropicales. FAO Documento Técnico de Pesca. No. 234. Roma, Italia. 
Pinto, M., M. Ximenes \& A. Fonteles. 2004. Idade e crescimento do badejo-mira, Mycteroperca rubra (Bloch 1793), no sudeste do Brasil. Arquivos de Ciências do Mar 37: 7-14.

Powell, D. 1979. Estimation of mortality and growth parameters for the length frequency of a catch Report process-V Réunion Conseil International pour L'Exploitation de la Mer. 175: 167-169.

Ricker, W. 1975. Computation and interpretation of biological statistic of fish population. Department of Environmental Fisheries and Marine Service. Bull. Fish. Res. Board Can. 191:1-382

Rodríguez, J. 1985. Reproducción del chere-chere, Haemulon steindachneri (Jordan y Gilbert 1982) (Perciformes: Haemulidae). Tesis de Grado, Licenciatura en Biología, Universidad de Oriente, Cumaná, Venezuela.

Romero, M. \& L.W. González. 1986. Edad y crecimiento de la mojarra, Eugerres plumieri (Cuvier en C \& V 1830) (Pisces: Gerreidae), de la laguna de la Restinga, Isla de Margarita, Venezuela. Bol. Inst. Oceanogr. Venezuela. 25: 21-34.

Servicio de Meteorología de la Fuerza Aérea Venezolana. 1990-2006. Sistema CLICOM, resumen climatológico, estación Porlamar, indicativo int. 80421, Serial Nacional 0871. Porlamar, Venezuela.
Sparre, P. \& S. Venema. 1997. Introducción a la evaluación de recursos pesqueros tropicales-Parte 1: manual. FAO Documento Técnico de Pesca No. 306. Roma, Italia.

Tavares, R. 2001. Estudio sobre la biodiversidad de tiburones en el parque nacional Archipiélago Los Roques. Informe técnico final para la oficina de diversidad biológica del Ministerio del Ambiente y de Los Recursos Naturales. Fundación Científica Los Roques, Venezuela.

Taylor, C. 1958. Cod growth and temperature. Journal of Conseil International pour L'Exploitation de la Mer. 23: 366-370.

Tolentino, V. \& C. Mendoza. 2001. Edad y crecimiento del tiburón martillo Sphyrna lewini (Griffith \& Smith 1834) en el Pacífico central de México. Ciencias Marinas 27: 501-520.

Tresierra, A. \& Z. Culquichicon. 1993. Biología pesquera. Libertad, Trujillo, Perú.

Tresierra, A., Z. Culquichicon \& B. Veneros. 1995. Dinámica de poblaciones de peces. Libertad, Trujillo, Perú.

Walford, L. 1946. A new graphic method of describing the growth of animals. Bull. Mar. Biol. 90: 141-147.

Wetherall, J. 1986. A new method for estimating growth and mortality parameters from length-frequency data. Fishbyte 4: 12-14. 